\title{
PERTool - Uma Ferramenta Educacional para Manipulação de Redes PERT/CPM
}

\author{
Paulo Henrique Lima Oliveira ${ }^{1}$, João Darcy T. Sant'Anna', \\ Flaviane Vicente T. Sant'Anna ${ }^{1}$, Diego Bevilaqua ${ }^{1}$, \\ Ana Carolina G. Inocêncio ${ }^{1}$, Heitor Costa ${ }^{2}$, Paulo A. Parreira Júnior ${ }^{2}$ \\ ${ }^{1}$ Instituto de Ciências Exatas - Universidade Federal de Goiás - Regional Jataí - \\ Caixa Postal 03 - 75801-615 - Jataí-GO - Brasil \\ ${ }^{2}$ Departamento de Ciência da Computação - Universidade Federal de Lavras \\ Caixa Postal 3.037 - 37.200-000 - Lavras - MG - Brasil \\ \{ph.lima.tecnico, diego.bevilaqua86, jdtsncomp, flaviane.vicente, \\ anacarolina.inocencio\}@gmail.com, \{heitor, pauloa.junior\}@dcc.ufla.br
}

Resumo. Professores, estudantes e gerentes de projetos relacionados aos cursos de Ciência da Computação, Engenharias e áreas afins têm relatado dificuldades no ensino-aprendizagem de alguns conteúdos utilizando recursos pouco interativos como aulas expositivas baseada em livros e apresentações com slides, além da onerosa replicação dos exemplos apresentados em sala sem o auxilio de ferramentas computacionais. Este artigo apresenta a PERTool, uma ferramenta educacional digital que permite ao usuário criar e manipular redes PERT/CPM, a qual é um dos assuntos abordados em disciplinas como Pesquisa Operacional e Projeto de Software. Esta técnica é utilizada para otimizar cronogramas de tempo e custo durante o desenvolvimento de variados tipos de projetos.

\section{Cenário de uso}

\subsection{Introdução}

Redes PERT/CPM (Program Evaluation and Review Technique/Critical Path Method) são técnicas utilizadas para auxiliar no planejamento, no agendamento, no monitoramento e no controle das atividades de um projeto que estejam relacionados entre si [1]; esse conjunto de atividades é comumente conhecido como cronogramação [3]. No contexto de ensino na área de Computação, essas atividades são apresentadas em disciplinas relacionadas à Engenharia de Software; mais especificamente, quando o assunto é a construção e o acompanhamento de cronogramas de projetos de software. A gerência de projetos é uma atividade essencial e complexa [4], pois o bom gerenciamento não garante o sucesso de um projeto, mas o mau gerenciamento aumenta significativamente as chances de um projeto falhar. Um projeto de sucesso é finalizado no prazo e no orçamento planejados, gerando um produto de qualidade com uma equipe motivada ao final do projeto [4].

No âmbito do gerenciamento de projetos de software, muitas vezes a precisão nos cronogramas é mais importante do que a precisão nos custos, uma vez que o não cumprimento do cronograma pode [4]: (i) reduzir o impacto de um produto/serviço no mercado; (ii) provocar insatisfação dos clientes; e (iii) gerar problemas de integração 
entre sistemas. Por exemplo, uma equipe de meteorologistas brasileiros pode deixar de realizar experimentos em um período propício para observação da Amazônia, caso o software para análise das imagens de satélite não seja entregue até o mês de setembro, quando as chuvas e a nebulosidade na região diminuem [13]. Sendo assim, ressalta-se a importância do correto entendimento dos conceitos de cronogramação, em especial, das redes PERT/CPM, por parte dos alunos e futuros profissionais. Tal importância é corroborada pela incidência desse assunto em questões recentes das provas de avaliação de desempenho acadêmico no Brasil, como o ENADE (Exame Nacional de Desempenho de Estudantes) - provas de 2011 e de 2014 [11, 12].

Uma ferramenta educacional digital é um conjunto de recursos computacionais projetados com a intenção de serem utilizados no contexto do processo de ensinoaprendizagem [2]. A simulação é um recurso utilizado por este tipo de software e consiste em atividades que colocam o aluno diante do computador como manipulador de situações que "imitam" ou aproximam-se do cenário real. O uso de ferramentas educacionais digitais pode trazer alguns benefícios para o processo de ensinoaprendizagem [5], como por exemplo:

- Os alunos podem relacionar de forma mais adequada o conteúdo teórico, aprendido nas disciplinas, com a prática;

- A interatividade proporcionada por esse tipo de ferramenta pode facilitar o aprendizado de conceitos relacionados a características dinâmicas e/ou abstratas de um sistema;

- A flexibilidade proporcionada por esse tipo de recurso educacional permite que os alunos testem novas entradas para determinado problema e vejam os resultados em tempo real, melhorando a interatividade entre aluno e conceito ensinado; e

- O aluno pode continuar estudando e experimentando novas configurações de entrada/saída para determinado problema fora do ambiente de sala de aula, o que não é tão simples de ser realizado com a utilização de outras ferramentas pouco interativas, tais como, livros-textos, apresentações de slides ou vídeo-aulas.

Assim, neste trabalho, o objetivo é trazer os benefícios comentados anteriormente para a área de ensino de cronogramação. Para isso, foi desenvolvida uma ferramenta educacional para construção e manutenção de redes PERT/CPM, denominada PERTOOl. O diferencial oferecido por essa ferramenta é o seu enfoque educacional, haja vista que as demais ferramentas existentes para esse tipo de conteúdo $[7,8,12]$, comentadas na Seção 3 deste trabalho, não possuem esse enfoque. Além disso, outro aspecto que ressalta a importância da PERTOOl é o fato de ela ser opensource, uma vez que a maioria das ferramentas para se trabalhar com redes PERT/CPM são plug-ins para ferramentas proprietárias, tais como, MS Project. Por ser open-source, o acesso à PERTOOl por professores e por alunos é facilitado, bem como a contribuição de pesquisadores e desenvolvedores para seu aprimoramento.

O restante deste trabalho está organizado da seguinte forma. Na Seção 2, são apresentados conceitos básicos sobre redes PERT/CPM. Na Seção 3, são descritos alguns trabalhos relacionados. Na Seção 4, é apresentada a ferramenta PERTool, descrevendo suas funções, $\mathrm{Na}$ Seção 5, são discutidos o planejamento e os resultados 
obtidos da avaliação realizada com alunos de graduação em computação sobre a ferramenta PERTOOl. Na Seção 6, estão presentes as considerações finais e sugestões de trabalhos futuros.

\subsection{Redes PERT/CPM}

Redes PERT/CPM são representações gráficas de um cronograma, nas quais são apresentadas a sequência lógica $\mathrm{e}$ as interdependências entre as tarefas desse cronograma. Essas redes são compostas por atividades e eventos [1] em um grafo orientado. Uma atividade compõe uma aresta do grafo, sendo a execução efetiva de uma operação, a qual representa o consumo de tempo e/ou recursos no projeto e possuem duração, podendo estar relacionadas com outras atividades. Um evento corresponde ao vértice do grafo, sendo um marco dentro da rede, representando o início e/ou o fim de uma atividade, e não consome tempo nem recursos em um projeto.

Exemplos de atividades e eventos são apresentados na Figura 1 (todas as figuras apresentadas neste trabalho foram geradas com o auxílio da ferramenta PERTOOl). Por exemplo, a atividade "Pintar porta" é representada com uma seta (aresta direcionada) ligando dois eventos (dois nós), sendo o evento 1 o marco de início da atividade e o evento 2 o marco de fim da atividade. Pode haver dependências entre as atividades de uma rede PERT/CPM, como apresentado na Figura 2. Nesse caso, é possível perceber que a atividade " $\mathrm{B}$ " só poderá ser iniciada quando a atividade " $\mathrm{A}$ " for terminada, pois o evento 2, que corresponde ao início da atividade "B", também é o evento final da atividade "A". Nesse caso, diz-se que as atividades "A" e "B" são executadas sequencialmente.

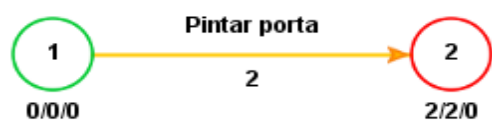

Figura 1 - Exemplo de uma Atividade e seu Evento Inicial e Final

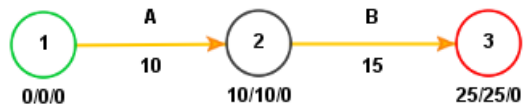

Figura 2 - Exemplo de Dependência entre Atividades

Há atividades que podem ser executadas em paralelo, conforme apresentado na Figura 3. Nessa figura, as atividades " $A$ " e "B" podem ser executadas ao mesmo tempo, pois existem duas setas saindo de um mesmo evento de início (evento 1), caracterizando o paralelismo entre elas. Além disso, há atividades chamadas "atividades fantasmas", um tipo especial de atividade que não consome tempo nem recursos. Atividades fantasmas são representadas por uma linha pontilhada (Figura 4). Um dos casos em que uma atividade fantasma deve ser utilizada é para mostrar a interdependência entre múltiplas atividades. Por exemplo, na Figura 4, a atividade "Café com leite" depende das atividades "Moer café" e "Comprar leite", porém a atividade "Coalhada" depende apenas da atividade "Comprar leite".

Um caminho de uma rede PERT/CPM é uma rota que segue as setas do evento inicial ao evento final. O comprimento de um caminho é a soma das durações das atividades desta rota; nos exemplos das Figuras 1 à 4, a duração é apresentada abaixo do nome das atividades. O caminho com maior comprimento é denominado caminho crítico da rede, pois as atividades sobre esse caminho são críticas, ou seja, são as atividades que consomem a maior quantidade de recursos do projeto. O caminho crítico é representado nas figuras anteriores pelas linhas de cor laranja. 


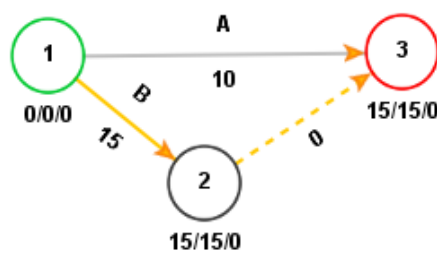

Figura 3 - Exemplo de Atividades Executadas em Paralelo

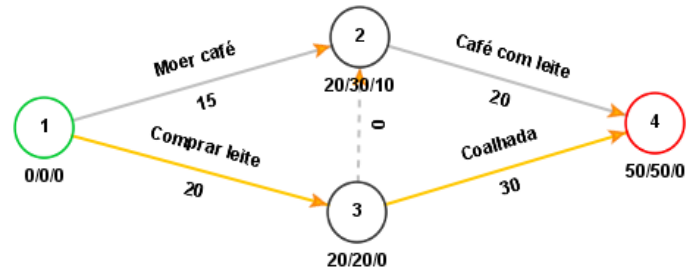

Figura 4 - Exemplo de interdependência entre Atividades

Uma das características interessantes das redes PERT/CPM é o cálculo do tempo de execução. O tempo de execução de uma rede PERT/CPM está relacionado com os conceitos de tempo cedo, tempo tarde e folga. O tempo cedo é o tempo necessário para um evento ser atingido, sem atrasos. Para cada evento da rede, o tempo cedo é calculado por tempo cedo = máximo (tempo cedo do evento anterior + duração). $\mathrm{O}$ tempo tarde corresponde ao tempo limite para atingir um evento (deadline), de modo que não haja atrasos no projeto. Para cada evento da rede, o tempo tarde é calculado por tempo tarde = mínimo (tempo tarde do evento posterior - duração). A folga corresponde à diferença entre o tempo tarde e o tempo cedo e é calculada por folga $=$ (tempo tarde - tempo cedo). As informações colocadas abaixo dos eventos nas redes das figuras anteriores descrevem o tempo cedo/tempo tarde/folga de cada evento, respectivamente.

\section{Desenvolvimento e Apresentação do Software}

Nesta seção, são descritas as principais funções existentes na ferramenta PERTOOl, destacando as diferenças entre os demais softwares analisados. Além disso, à medida que as funções são descritas, discussões a respeito dos benefícios educacionais que podem ser obtidos com a utilização dessa ferramenta são apresentados. PERTool é uma ferramenta gráfica, desenvolvida em Java, que pode ser obtida gratuitamente por meio de download e cujo código está disponível em um repositório de acesso público: https://gitlab.com/joaodarcy/perttool.

A internacionalização (internationalization - i18n) do conteúdo é uma característica positiva e importante da $\mathrm{PERTOOl}$, pois a falta de material para estudo no idioma nativo do estudante pode tornar-se uma barreira para o entendimento do conteúdo abordado. Dessa forma, o aluno deve superar as barreiras naturais impostas pelo aprendizado e as barreiras linguísticas, a qual nem sempre é superada de forma fácil por todos. Apesar das demais ferramentas contemplarem essa característica, elas não possuem material didático sobre redes PERT/CPM internacionalizado. O recurso de i18n foi implementado na PERTOOl, disponibilizando rótulos na interface gráfica e material didático sobre redes PERT/CPM em três idiomas (Português, Inglês e Espanhol) (Figura 5 - A). No material didático disponível na PERTOOl, foram disponibilizados um tutorial sobre a técnica PERT/CPM, um manual de uso da ferramenta e um conjunto de três redes de exemplo (internacionalizadas). Essa característica é positiva, pois torna PERTOOl acessível a maior quantidade de pessoas, minimizando a barreira linguística como fator limitante ao aprendizado sobre os conceitos abordados. 


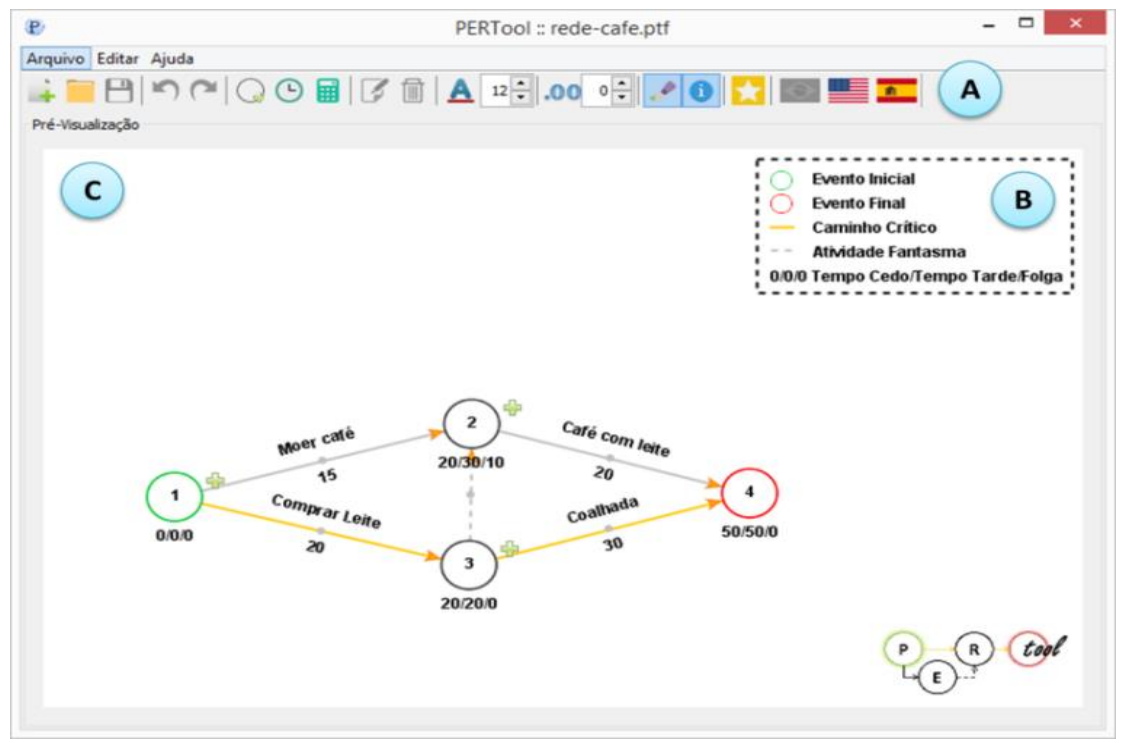

Figura 5 - Interface Gráfica da Ferramenta PERTool

Em PERTOOl, são oferecidos recursos para auxiliar alunos e professores a criarem visualizações de redes PERT/CPM para utilização em trabalhos acadêmicos, slides, vídeo-aulas, entre outros. Para isso, em PERTool, é disponibilizada a função de exportação das redes criadas para imagens com formato PNG (Portable Network Graphics). Além disso, com a PERTOOl, os usuários podem salvar (armazenar persistentemente) seu trabalho em um arquivo com extensão .ptf, sendo interessante, pois permite ao usuário (i) continuar seu trabalho posteriormente; e (ii) compartilhar o seu projeto com outras pessoas, tornando o trabalho de construção de redes PERT/CPM colaborativo. As imagens utilizadas na Seção 2 deste artigo foram exportadas com PERTOOl.

Outro recurso importante na PERTOOl, não disponível nas demais ferramentas analisadas, é as legendas explicativas sobre os elementos gráficos de uma rede PERT/CPM (Figura 5 - B), as quais podem ser desabilitadas pelo usuário. Essas legendas contribuem de forma positiva do ponto de vista educacional, pois permitem ao usuário relembrar os principais conceitos durante a construção de suas redes.

Realizar os cálculos de tempo da rede (tempo cedo, tempo tarde e folga) manualmente é um dos desafios ao se confeccionar uma rede PERT/CPM por ser onerosos e suscetíveis a falhas. Dessa forma, pode ser vantajoso utilizar PERTOOl, visto que esses cálculos são realizados automaticamente, à medida que usuário constrói sua rede (Figura 5 - C). Esse é um ponto positivo, pois o usuário consegue identificar quais são os gargalos e os atrasos, ao montar a sua rede e em tempo real. $\mathrm{Na}$ ferramenta PERTOOl, o caminho crítico da rede é calculado à medida em que a mesma é construída pelo usuário (setas de cor laranja da Figura 5 - C). Isto é interessante, pois o usuário consegue saber, visualmente, quais são os caminhos que consomem mais recursos, sendo uma informação valiosa no decorrer do projeto de software. Ao criar um cronograma de um projeto de software, deve-se começar com um conjunto de tarefas [4]. Se forem utilizadas ferramentas automáticas, a subdivisão do trabalho entra como uma rede ou resumo de tarefas. Dados de esforço, de duração e de data de início são definidos para cada tarefa. Além disso, as tarefas podem ser atribuídas a indivíduos 
específicos. Com base nessas informações, é gerado um gráfico de Gantt, utilizado para ilustrar as diferentes etapas de um projeto de software, permitindo uma análise do progresso conforme o tempo de execução do projeto. Esse gráfico pode ser exportado como uma imagem no formato PNG em PERTOOl.

\section{Considerações finais}

Nesta seção, é discutida a avaliação realizada com a ferramenta PERTool, descrevendo o método utilizado, os resultados obtidos e as análises feitas.

\subsection{Metodologia de Avaliação}

A avaliação de PERTOOl foi conduzida de acordo com um modelo de avaliação de jogos educacionais proposto por Savi et al. [15]. Uma das vantagens desse modelo é não consumir muito tempo em sua aplicação, uma vez que, em geral, não há tempo hábil para longas sessões de avaliações nas disciplinas. Com esse modelo, a ferramenta é avaliada para saber se: (i) consegue motivar os alunos a utilizarem o recurso como material de aprendizagem; (ii) proporciona boa experiência nos usuários; e (iii) gera percepção de utilidade educacional (se os alunos acham que a ferramenta é útil e sabem utilizá-la).

Algumas adaptações foram realizadas neste modelo para adequá-lo ao contexto de uma ferramenta educacional. Algumas questões foram adaptadas/excluídas para a avaliação de PERTOOl, por tratarem de elementos específicos de jogos educacionais digitais, os quais não eram adequados ao contexto de ferramenta educacional. Para avaliação, foi proposto um questionário com o intuito de coletar dados dos entrevistados sobre os parâmetros que compõem o modelo de avaliação proposto por Savi [15]. O questionário é composto por 34 afirmações (Tabela 2), para as quais os participantes da avaliação deveriam indicar o quanto concordam com as mesmas, de acordo com uma escala Likert de 7 pontos variando de "discordo fortemente" a "concordo fortemente".

\subsection{Análise dos Resultados}

A avaliação da ferramenta PERTOOl foi realizada por 18 alunos do curso Bacharelado em Ciências da Computação da Universidade Federal de Goiás - Regional Jataí (graduação incompleta). Os participantes cursavam a disciplina de Projeto de Software, os quais estudavam sobre cronogramação, incluindo redes PERT/CPM. Para essa avaliação, foi ministrado um treinamento em sala de aula para relembrar os conceitos sobre redes PERT/CPM e apresentar aos alunos as funções de PERTOOl. Durante a avaliação de PERTool, cada aluno a utilizou por cerca de 30 minutos, nos quais os alunos deveriam montar algumas redes PERT/CPM propostas pela equipe que conduziu o experimento. Em seguida, os alunos responderam ao questionário de avaliação apresentado na Tabela 2. Por questão de espaço, as respostas dadas pelos participantes foram omitidas, apresentando-se apenas os resultados por categoria (M - Motivação, E Experiência do Usuário e A - Aprendizagem). Contudo, a planilha completa com os resultados pode ser visualizada em https://goo.gl/vaydP5.

As dimensões da categoria "Motivação" são Atenção (perguntas 1 e 2 da Tabela 2), Relevância (perguntas 3 à 7), Confiança (perguntas 8 à 14) e Satisfação (perguntas 12 à 15). De acordo com os resultados da Tabela 3 , a avaliação mostrou que houve forte concordância por parte dos avaliadores com relação à Atenção $(83,3 \%)$ e Confiança 
V Congresso Brasileiro de Informática na Educação (CBIE 2016)

Anais dos Workshops do V Congresso Brasileiro de Informática na Educação (CBIE 2016)

(88,9\%). A Atenção refere-se às respostas cognitivas dos alunos a estímulos racionais. A Confiança refere-se à criação de expectativas positivas nos alunos, ao proporcionar-lhes experiências de sucesso decorrentes de suas próprias habilidades e esforço [15].

Tabela 2 - Perguntas do Questionário de Avaliação

\begin{tabular}{|c|c|c|}
\hline \# & Perguntas & Categoria \\
\hline 1 & Houve algo interessante na ferramenta que capturou minha atenção. & $\mathrm{M}$ \\
\hline 2 & O design da ferramenta é atraente. & M \\
\hline 3 & Ficou claro para mim como o conteúdo da ferramenta está relacionado com coisas que eu sabia. & $\mathrm{M}$ \\
\hline 4 & Eu gostei tanto da ferramenta que gostaria de aprender mais sobre o assunto abordado por ela. & M \\
\hline 5 & O conteúdo abordado na ferramenta é relevante para meus interesses. & $\mathrm{M}$ \\
\hline 6 & Eu poderia relacionar o conteúdo do jogo com coisas que já vi, fiz ou pensei. & $\mathrm{M}$ \\
\hline 7 & O conteúdo abordado na ferramenta será útil para mim. & $\mathrm{M}$ \\
\hline 8 & A ferramenta foi mais fácil de entender do que eu imaginava. & M \\
\hline 9 & Foi fácil identificar e lembrar dos pontos importantes do conteúdo na ferramenta. & M \\
\hline 10 & O conteúdo da ferramenta foi exposto de forma tão interessante que foi difícil tirar a atenção dele. & $\mathrm{M}$ \\
\hline 11 & Eu consegui entender boa parcela do material da ferramenta. & $\mathrm{M}$ \\
\hline 12 & Realizar minhas tarefas na ferramenta me deu um sentimento de realização. & $\mathrm{M}$ \\
\hline 13 & Eu aprendi algumas coisas com a ferramenta positivamente surpreendentes ou inesperadas. & M \\
\hline 14 & Os textos de feedback ou outros comentários da ferramenta me ajudaram. & $\mathrm{M}$ \\
\hline 15 & Eu me senti bem ao completar minhas tarefas na ferramenta. & M \\
\hline 16 & Eu não percebi o tempo passar enquanto usava a ferramenta. & $\mathrm{E}$ \\
\hline 17 & Eu perdi a consciência do que estava ao meu redor enquanto usava a ferramenta. & $E$ \\
\hline 18 & Eu me senti mais no ambiente da ferramenta do que no mundo real. & $E$ \\
\hline 19 & Em nenhum momento, eu quis abandonar o uso da ferramenta. & $\mathrm{E}$ \\
\hline 20 & Eu me senti estimulado a aprender com a ferramenta. & $E$ \\
\hline 21 & Eu gostei da ferramenta e não me senti ansioso ou entediado. & $\mathrm{E}$ \\
\hline 22 & A ferramenta me manteve motivado a continuar utilizando-a. & $\mathrm{E}$ \\
\hline 23 & Minhas habilidades melhoraram gradualmente à medida que ia usando a ferramenta. & $\mathrm{E}$ \\
\hline 24 & Eu me senti bem-sucedido ao utilizar a ferramenta. & $E$ \\
\hline 25 & Eu alcancei rapidamente meus objetivos com a ferramenta. & $\mathrm{E}$ \\
\hline 26 & Eu me senti competente. & $E$ \\
\hline 27 & Eu senti que estava tendo progresso à medida que ia usando a ferramenta. & $\mathrm{E}$ \\
\hline 28 & Eu gostaria de utilizar esta ferramenta por mais tempo. & $E$ \\
\hline 29 & Quando interrompido, fiquei desapontado, pois tive que parar de usar a ferramenta. & $\mathrm{E}$ \\
\hline 30 & Eu usaria esta ferramenta novamente. & $\mathrm{E}$ \\
\hline 31 & Há poucas coisas na ferramenta que me irritaram. & $\mathrm{E}$ \\
\hline 32 & $\begin{array}{l}\text { Depois do uso da ferramenta, consigo lembrar de mais informações relacionadas ao tema } \\
\text { apresentado nela. }\end{array}$ & A \\
\hline 33 & Depois do uso da ferramenta, consigo compreender melhor os temas apresentados nela. & A \\
\hline 34 & Depois do uso da ferramenta, sinto que consigo aplicar melhor os temas relacionados com nela. & A \\
\hline
\end{tabular}

Além disso, a avaliação mostrou também que Satisfação (69,4\% das avaliações positivas) não obteve bons resultados quanto aos demais dessa categoria. Os alunos precisam ter sentimentos positivos sobre a experiência da aprendizagem e isso pode vir por meio de recompensas e reconhecimentos [15]. As propostas para melhorar os resultados desta dimensão podem vir com a gamificação, uma técnica que compreende a aplicação de elementos de jogos em atividades que não sejam jogos [14]. Por fim, a categoria "Motivação" obteve $80,9 \%$ de avaliações positivas, o que indica que o aluno se sente motivado ao utilizar a ferramenta educacional como auxilio para aprendizagem dos conteúdos sobre redes PERT/CPM.

A categoria "Experiência do Usuário" obteve, em média, 73,6\% das avaliações positivas, tendo como pior resultado a dimensão Desafio (afirmações 21 à 31 da Tabela 2 ), cujo valor foi de $69,4 \%$ de avaliações positivas. Esse resultado se deve ao fato de a mecânica dos jogos não estar aplicada à ferramenta PERTOOl. Para isso, como proposta de solução, também pode ser aplicada a gamificação para obter melhores resultados para essa categoria. Por fim, a categoria "Aprendizagem" obteve $74,1 \%$ das avaliações positivas, o que pode indicar a sedimentação dos conhecimentos do usuário utilizando PERTOOl. 
V Congresso Brasileiro de Informática na Educação (CBIE 2016)

Anais dos Workshops do V Congresso Brasileiro de Informática na Educação (CBIE 2016)

Tabela 3 - Média de Avaliações por Categoria

\begin{tabular}{|c|c|c|c|c|}
\hline \multirow{2}{*}{\multicolumn{2}{|c|}{ CATEGORIAS / DIMENSÕES }} & \multicolumn{3}{|c|}{ AVALIAÇÕES } \\
\hline & & Positivas (5 até 7 ) & Neutras (4 apenas) & Negativas (1 até 3 ) \\
\hline \multirow{4}{*}{ MOTIVAÇÃO } & Satisfação & $69,4 \%$ & $16,7 \%$ & $13,9 \%$ \\
\hline & Relevância & $81,1 \%$ & $10,0 \%$ & $8,9 \%$ \\
\hline & Confiança & $88,9 \%$ & $8,3 \%$ & $2,8 \%$ \\
\hline & Atenção & $83,3 \%$ & $13,9 \%$ & $2,8 \%$ \\
\hline \multicolumn{2}{|r|}{ MÉDIA } & $80,7 \%$ & $12,2 \%$ & $7,1 \%$ \\
\hline \multirow{3}{*}{$\begin{array}{l}\text { EXPERIÊNCIA } \\
\text { DO USUÁRIO }\end{array}$} & Desafio & $69,4 \%$ & $13,9 \%$ & $16,7 \%$ \\
\hline & Habilidade/Competência & $77,8 \%$ & $12,9 \%$ & $9,3 \%$ \\
\hline & MÉDIA & $73,6 \%$ & $13,4 \%$ & $13 \%$ \\
\hline APRENDIZAGEM & Aprendizagem & $74,1 \%$ & $20,4 \%$ & $5,7 \%$ \\
\hline
\end{tabular}

Além dessas questões, uma questão aberta foi disponibilizada para os avaliadores deixarem sua opinião sobre PERTool. Alguns desses comentários são "Ao aumentar o tamanho da fonte, deverá expandir também a distância dos eventos.", "Quando tento ligar dois eventos sucessivos em duas atividades ele não permite sabendo que o ideal seria criar uma fantasma, por conta de já saber disso falta orientação do software do porque isso acontece, e porque não criar a atividade fantasma automaticamente?" e "A ferramenta poderia auto ajustar a rede pert conforme fosse aumentando o tamanho das letras para que não ficasse desproporcional.”.

\section{Referências}

[1] H. S. Swanson, R. E. D. Woolsey (1969), “A PERT-CPM Tutorial”

[2] Sancho, J. M (1998) "Para Uma Tecnologia Educacional". Porto Alegre, Artmed.

[3] Lima, A et al. (2010) "Uma Ferramenta Baseada em Simulação Gráfica para Suporte ao Ensino de Redes de Computadores”. In Workshop de Educação em Informática Bahia-Alagoas-Sergipe (WEIBASE).

[4] Sommerville, "Engenharia de Software", 8 a edição, Editora Pearson.

[5] Judith S. Liebman, (1998) Teaching Operations Research: Lessons from Cognitive Psychology. Interfaces 28(2):104-110.

[6] Paas, F. G. W (1992) Training Strategies for Attaining Transfer of Problem-Solving Skill in Statistics: A Cognitive-Load Approach. Journal of Educational Psychology, Vol. 84, N. 4, 429434.

[7] WBS Schedule Pro, Disponível em: http://www.criticaltools.com/download.html. Acessado em: Abril/15.

[8] Visio PERT, Disponível em: http://goo.gl/PVEhGf. Acessado em: Abril/15.

[9] Critical Tools, Disponível em: http://www.criticaltools.com/. Acessado em: Abril/15.

[10] INEP - Instituto Nacional de Estudos e Pesquisas Educacionais Anísio Teixeira. ENADE 2014. Disponível em: http://portal.inep.gov.br/web/guest/enade/provas-e-gabaritos-2014. Acessado em: Abril/15.

[11] INEP - Instituto Nacional de Estudos e Pesquisas Educacionais Anísio Teixeira. ENADE 2011. Disponível em: http://portal.inep.gov.br/web/guest/enade/provas-e-gabaritos-2011. Acessado em: Abril/15.

[12] ProjectLibre, Disponível em: http://sourceforge.net/projects/projectlibre/. Acessado em: Abril/15.

[13] Jornal O Globo. "Falta de software empaca dados de satélite brasileiro". Disponível em: http://goo.gl/3koFCr. Acessado em: Abril/15.

[14] Fadel, Ulbricht, Batista, Vanzin, "Gamificação na educação” (2014), Editora Pimenta Cultural.

[15] Savi, R., Von Wangenheim, C. G., Ulbricht, V., and Vanzin, T. (2010) "Proposta de um Modelo de Avaliação de Jogos Educacionais". In: RENOTE - Revista Novas Tecnologias na Educação, vol. 8 , no. 3 . 